\title{
Efficient Coupling of Monte Carlo and Level Set Methods for Topography Simulation
}

\author{
O. Ertl, C. Heitzinger, and S. Selberherr \\ Institute for Microelectronics, TU Wien, 1040 Vienna, Austria \\ \{ertl|heitzinger|selberherr\}@iue.tuwien.ac.at
}

\begin{abstract}
We have developed a topography simulation method which combines advanced level set techniques for surface evolution with Monte Carlo flux calculation. The result is an algorithm with an overall complexity and storage requirement scaling like $O(N \log N)$ with surface disretization. The calculation of particle trajectories is highly optimized, since spatial partitioning is used to accelerate ray tracing. The method is demonstrated on $\mathrm{Si}$ etching in $\mathrm{SF}_{6} / \mathrm{O}_{2}$ plasma.
\end{abstract}

\section{Introduction}

In the past numerous techniques for tracking surfaces, calculating etching and deposition rates, and their application to topography simulation were reported [1]. We present an efficient and accurate way for coupling the level set (LS) method with Monte Carlo (MC) flux calculation. Both methods are most promising for large three-dimensional topography simulation, since their numerical efforts scale almost linearly with surface discretization, if realized using narrow band and efficient ray-tracing techniques.

\section{Surface Evolution}

To overcome the difficulties in surface evolution, which appeared especially in three dimensions, the LS method was developed [2]. This method represents the surface as the root of a function $\Phi$ and describes its time evolution by

$$
\frac{\partial \Phi}{\partial t}+F|\nabla \Phi|=0
$$

where $F$ denotes the surface velocity. Originally the LS function was stored on the whole space. To save calculation time, the narrow band and - a further development - the sparse field method were introduced $[2,3]$. To decrease storage requirements as well, tree data structures were used [4]. We use the sparse field method in combination with a map, indexed by the coordinates, to access all active grid points. In this way complexity and storage requirement of the surface evolution algorithm are reduced down to order $O(N \log N)$, where $N$ is the number of surface elements. After each time step the actual surface is extracted by the marching cubes algorithm [5]. 


\section{Flux Calculation}

To determine the local etching and deposition rates in topography simulations the fluxes and influence of incident particles have to be determined. Assuming ballistic transport the flux distribution $\Gamma(\mathbf{x}, \mathbf{t})$ at point $\mathbf{x}$ of the surface $S$ in dependence of the incident direction $\mathbf{t}$ is described by

$$
\Gamma(\mathbf{x}, \mathbf{t})=\Gamma_{d}(\mathbf{x}, \mathbf{t})+\int_{S} \frac{v i s\left(\mathbf{x}, \mathbf{x}^{\prime}\right)}{\left\|\mathbf{x}-\mathbf{x}^{\prime}\right\|^{2}} \int R\left(\mathbf{t}, \mathbf{t}^{\prime}\right) \Gamma\left(\mathbf{x}^{\prime}, \mathbf{t}^{\prime}\right) d \Omega^{\prime} d A^{\prime} .
$$

$\Gamma_{d}$ represents the direct flux from the source, while the integral term incorporates the reemission of particles. The function $v i s\left(\mathbf{x}, \mathbf{x}^{\prime}\right)$ is 1 or 0 , if point $\mathbf{x}$ and $\mathbf{x}^{\prime}$ are in line of sight or not, respectively. $R\left(\mathbf{t}, \mathbf{t}^{\prime}\right)$ is the probability distribution for an incoming particle of direction $\mathbf{t}$, that it is reemitted in direction $\mathbf{t}^{\prime}$. Assuming isotropic reemission (2) can be solved by direct integration [6]. However, this approach reaches its limits when considering higher order reemission, specular reemission, or energy dissipation. Furthermore, it is restricted to small simulation domains, because the complexity for calculating the integral term is of order $O\left(N^{2}\right)$. Additionally, the visibility check has to be performed. The MC method is an alternative approach. Many particle trajectories are simulated and their contributions to the surface velocity dependent on their types, energies, and incidence directions are summed up. The major task is the calculation of ray/surface intersections. To reduce the effort of tracking one particle down to order $O(\log N)$, we use spatial subdivision with a $k d$-tree as shown in Figure 1. The dividing planes always correspond to grid planes to utilize the property of the marching cubes algorithm that extracted surface elements always lie within just one grid cell. To provide fast traversal times, the usage of neighbour-links trees has been proposed [7]. Instead of trees we use arrays to further speed up neighbour access. It can be shown that the storage requirement of these additional neighbour-links arrays is of order $O(N \log N)$. To keep statistical accuracy constant, the number of simulated particles must be of order $O(N)$, leading to an overall numerical complexity of $O(N \log N)$. Figure 2 depicts the scaling law of particle tracking times and storage requirements for a two-dimensional model of a trench as shown in Figure 1. As additional enhancement, we assign all particles a volume as decribed in [8]. If a particle hits the surface, its volume is decreased corresponding to the sticking probability, before the calculation of its trajectory is continued. In this way several events are raised on the surface by each particle, which improves the statistics, especially for low sticking probabilities.

\section{MC-LS Coupling}

Common surface extraction techniques as the marching cubes algorithm [5] lead to large variations in surface element sizes. To reduce statistical errors averaging is obligatory. Until now combined MC calculations were coupled to LS by calculating the surface velocity for each element first and subsequent extension to all grid points within the narrow band [9]. This two-step procedure leads to unnecessary loss of information. We map the flux information of each individual particle directly to grid points surrounding the impact location. Averaging is carried out for each grid point $P$ by considering all incidences within a neighbourhood $B_{\varepsilon}\left(P^{\prime}\right)$ of radius $\varepsilon$ around the closest surface point 


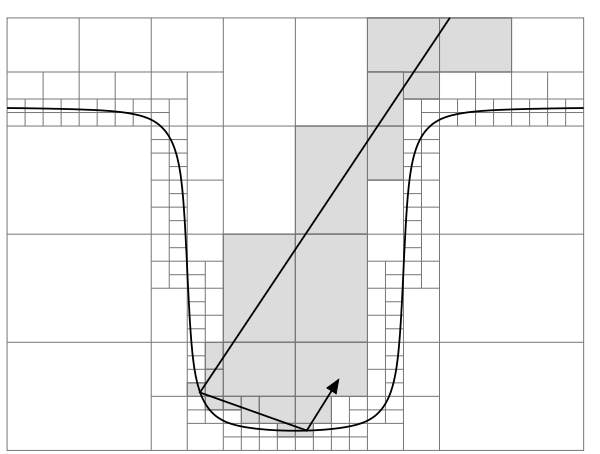

Figure 1: Spatial subdivision is used for efficient particle tracking. For each box all neighbours are stored in a pointer array to provide fast traversals.

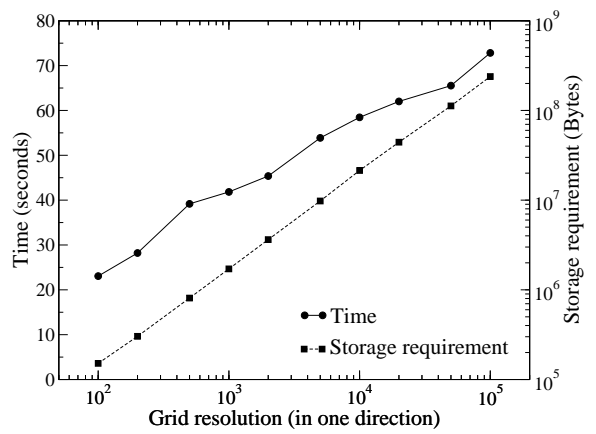

Figure 2: The required time for tracking 3 million particles and the storage requirement for the $k d$-tree are given for different grid resolutions. Calculations were performed on a $2.8 \mathrm{GHz}$ Pentium4.

$P^{\prime}$ of the grid point $P$ (Figure 3 ). The flux contributions of each particle are weighted by

$$
w_{\varepsilon}(P, \mathbf{t})=\frac{\min \left(\mathbf{t} \cdot \mathbf{n}_{P^{\prime}}, 0\right)}{\int_{B_{\varepsilon}\left(P^{\prime}\right)} \min (\mathbf{t} \cdot \mathbf{n}, 0) d A}
$$

where $\mathbf{t}$ denotes the particle direction and $\mathbf{n}_{\left(P^{\prime}\right)}$ the surface normal vector (at $P^{\prime}$ ). The denominator can be precalculated for directions within a certain cone around $-\mathbf{n}_{P^{\prime}}$, so that the evaluation of the integral is rarely necessary. If combined with the sparse field or narrow band LS method, the additional numerical effort is moderate. In return, no additional extension of the surface velocity is required.

\section{Example: Si Etching in $\mathrm{SF}_{6} / \mathrm{O}_{2}$ Plasma}

We applied our combined LS-MC method to the model of $\mathrm{Si}$ etching in $\mathrm{SF}_{6} / \mathrm{O}_{2}$ plasma given in [10]. Therein coverages for the neutrals are introduced to describe the site balance following a Langmuir-Hinshelwood type model. Our numerical framework supports the transformation of surface coverages to the next time step, because their values are calculated on each grid point and not on surface elements. The coverages for surface points are determined by linear interpolation. In our calculation energy dissipative reflections of ions and coverage dependent sticking probabilities for neutrals are fully taken into account. Different materials are incorporated by assigning them to grid points based on the location of the closest surface points. Figure 4 shows crosscuts of trenches for different $\mathrm{SF}_{6} / \mathrm{O}_{2}$ flux ratios.

\section{Conclusion}

Our proposed method enables MC-LS topography simulations in a numerically efficient manner. The presented techniques are well suited for large three-dimensional simula- 


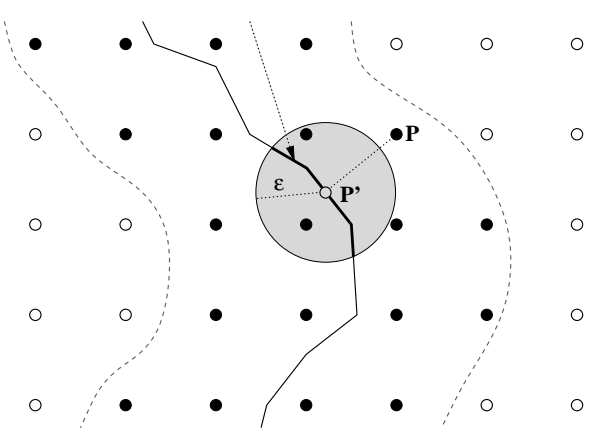

Figure 3: For each grid point $\mathrm{P}$ within the narrow band (dashed) the corresponding closest surface point $\mathrm{P}^{\prime}$ is determined. Each particle (arrow) hitting the surface (solid) within a certain neighbourhood of $\mathrm{P}$ ' contributes to the surface velocity of $\mathrm{P}$.

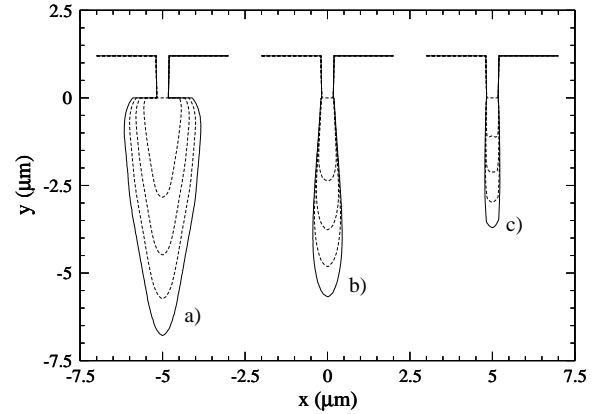

Figure 4: $\quad \mathrm{SF}_{6} / \mathrm{O}_{2}$ plasma etching crosscuts of $0.35 \mu \mathrm{m}$ trenches after $300 \mathrm{~s}$ for different $\mathrm{SF}_{6} / \mathrm{O}_{2}$ flux ratios: a) $80 \mathrm{sccm} / 0 \mathrm{sccm}$, b) $45 \mathrm{sccm} / 35 \mathrm{sccm}$, c) $30 \mathrm{sccm} / 50 \mathrm{sccm}$. Parameters were taken from [10].

tions. In contrast to direct integration methods for flux calculation, the MC method is not restricted to approximations of the flux distribution equation (2) and also incorporates general energy and arbitrary direction distributions. Additionally, the flux calculation can be straightforwardly parallelized on multi-CPU/core systems.

\section{References}

[1] U.H. Kwon, W.-J. Lee, "Three dimensional deposition topography simulation based on new combinations of flux distribution and surface representation algorithms", Thin Solid Films, vol. 445, pp. 80-89, 2003.

[2] J.A. Sethian, "Level Set Methods and Fast Marching Methods", Cambridge University Press, 1999.

[3] R.T. Whitaker, "A level-set approach to 3D reconstruction from range data", J. Comp. Vision, pp. 203-231, vol. 29, 1998.

[4] J. Strain, J. Comp. Phys., "Tree methods for moving interfaces", vol. 151, pp. 616-648, 1999.

[5] W. Lorensen, H. Cline, "Marching cubes: a high resolution 3D surface construction algorithm.”, Comput. Graph., vol. 21, pp. 163-169, 1987.

[6] C. Heitzinger et. al, "Feature-scale process simulation and accurate capacitance extraction for the backend of a 100-nm aluminum/TEOS process", IEEE Trans. Electron Devices, vol. 51, pp. 1129-1134, 2004.

[7] V. Havran, "Heuristic Ray Shooting Algorithms", PhD thesis, Czech Technical University, Prague, 2000.

[8] T. Smy, S.K. Dew, R.V. Joshi, "Efficient modeling of thin film deposition for low sticking using a three-dimensional microstructural simulator", J. Vac. Sci. Technol. A, vol. 19, pp. 251-261, 2001.

[9] B. Radjenovic, J.K. Lee, "3D feature profile evolution simulation for $\mathrm{SiO}_{2}$ etching in fluorocarbon plasma", Proc. $17^{\text {th }}$ ICPIG, 17/142, 2005.

[10] R.J. Belen et al., "Feature-scale model of Si etching in SF6/O2 plasma and comparison with experiments", J. Vac. Sci. Technol. A, vol. 23, pp. 1430-1439, 2005. 